\author{
猫运动皮层神经元和 S100、GFAP 阳性细胞的 \\ 年龄相关性变化 \\ 申 林, 孙庆艳, 考传超, 华田苗* \\ (安徽师范大学 生命科学学院, 安徽 芜湖 241000)
}

摘要: 比较了青、老年猫运动皮层神经元与S100、GFAP免疫阳性胶质细胞的形态学变化, 并探讨其与衰老过程 中运动功能衰退的关系。采用Nissl染色显示青、老年猫运动皮层分层结构和神经元。免疫组织化学方法 (SABC法) 显 示青、老年猫运动皮层S100免疫反应阳性(S100-immunoreactive, S100-IR)细胞及胶质纤维酸性蛋白免疫反应阳性 (GFAP-immunoreactive, GFAP-IR)细胞。在Olympus 显微镜下, 用Moitcam 5000数码成像与分析系统计数运动皮层各层 神经元、S100-IR细胞及GFAP-IR细胞的数量, 并随机抽样测量S100-IR、GFAP-IR细胞的胞体直径。与青年猫相比, 老 年猫运动皮层 $\mathrm{V} 、 \mathrm{VI}$ 层神经元密度显著下降 $(P<0.01)$, 老年猫运动皮层中S100-IR 和GFAP-IR 细胞密度与胞体直径均显 著增加 $(P<0.01)$, 且细胞的免疫阳性反应较强。研究结果表明, 猫运动皮层的神经元密度在衰老过程中 V、VI层神经 元密度显著下降, 有可能会降低老年个体运动皮层对运动的调控能力; 随着衰老、运动皮层的星形胶质细胞出现明显 的反应性活化与增生，这对维持大脑运动皮层神经元的活性和神经元之间的通讯联系，从而延缓老年性运动功能衰退 具有重要意义。

关键词: 运动皮层; 星形胶质细胞; S100 蛋白; 胶质纤维酸性蛋白; 年龄相关变化

中图分类号: Q42 文献标识码: A 文章编号: 0254-5853-(2008)01-0056-07

\title{
Age-Related Changes in Neurons and S100-, GFAP-Immunoreactive Cells in the Motor Cortex of Cats
}

\author{
SHEN Lin, SUN Qing-yan, KAO Chuan-chao, HUA Tian-miao*
}

(College of Life Science, Anhui Normal University, Wuhu 241000, China)

\begin{abstract}
Morphological changes of neurons and S100-,GFAP-immunoreactive cells in the motor cortex of young adult and older cats were comparatively investigated, and the correlation of these changes and motor function degradation during senescence was discussed. Nissl staining was applied to show cortical layers and neurons. Immunohistochemical method was employed to exhibit S100-immunoreactive (S100-IR) and GFAP-immunoreactive (GFAP-IR) cells. Under an Olympus microscope, Moitcam 5000 Digital Image Acquisition and Analysis System was used to statistically count the number of total cortical neurons, GFAP-IR cells and S100-IR cells in each cortical layer, and the cell-body diameter of GFAP-IR cells and S100-IR cells were randomly sampled and measured. The density of total neurons in layer V and VI of the motor cortex showed a significant decrease in older cats $(P<0.01)$. Furthermore, the density and the cell-body diameter of GFAP-IR and S100-IR cells in the motor cortex of old cats were significantly increased when compared to that in young adults $(P<0.01)$. In addition, the S100 and GFAP immunoreactivity in the motor cortex of older cats was stronger than in younger ones. The density of total neurons in layer V and VI of the motor cortex was significantly reduced during aging, which might lead to a decreased motion mediation capacity of the motor cortex in older individuals. Moreover, with age, astrocytes in the motor cortex were significantly activated and increased, which is of great significance in maintaining normal neuronal activities, signalling between neurons and therefore slowing down age-dependent motor function degradation.
\end{abstract}

Key words: Motor cortex; Astrocyte; S100 protein; Glial fibrillary acidic protein; Age-related change

收稿日期：2007-09-17; 接受日期：2007-12-24

基金项目: 安徽省自然科学基金(070413138); 安徽省高校重点实验室科研基金; 安徽师范大学博士启动基金资助

“通讯作者 (Corresponding author), E-mail: tmhua@mail.ahnu.edu.cn

第一作者简介: 申林, 男(1977-), 汉族, 硕士研究生, E-mail: shenlin0558@126.com 
神经元是构成神经系统的结构和功能的基本 单位, 神经元之间以突触的方式相互联系, 从而完 成神经系统的总体功能, 因此神经元的年龄相关性 变化将会导致神经系统功能的相应变化。人及高等 动物复杂的运动行为和精细运动的执行受大脑运 动皮层的精确调控。有实验证明, 相对于年轻个体 而言, 老年个体对运动的调节和控制需要更多的大 脑皮层区域参与, 这是对老年个体运动皮层调节能 力下降的补偿 (Ward et al, 2006; Sailer et al, 2000)。 老年性大脑皮层神经元功能减退的原因目前尚不 很清楚, 现有研究结果显示, 年龄依赖性的皮层神 经元功能衰退可能与神经元自身的形态 (Dickstein et al, 2007)和调节能力（Komatsu et al，2006）改变 有关。胶质细胞广泛分布于神经元之间, 星形胶质 细胞作为中枢神经系统中数目占绝对优势的一类 胶质细胞, 具有多方面的生理意义, 对神经系统的 营养、支持、保护以及神经系统的发育、修复、再 生、免疫应答和神经元之间的通讯等方面都起着十 分重要的作用 (Kirchhoff et al, 2001; Jessen, 2004)。 衰老过程中中枢神经系统会出现胶质细胞反应性 变化, 有学者认为调控神经胶质细胞的基因的转录 随着衰老而增加（Erraji- Benchekroun et al, 2005）。 GFAP（glial fibrillary acidic protein）是一个 $56 \mathrm{kDa}$ 的中间丝蛋白,是区别星形细胞和其他胶质细胞一 个特异性的标志物。采用GFAP抗体标记星形胶质 细胞的分布和形态。S100蛋白是一种酸性钻结合蛋 白家族, 大多数存在于细胞内, 为细胞内钙受体蛋 白。S100蛋白家族大约有 16 个成员, 其中S100A和 S100B 是其主要成员。哺乳动物的中枢神经系统中 多以S100B形式存在。S100B蛋白是高度脑特异的, 具有神经保护作用, 可以提高神经元的存活率, 促 进胶质细胞发出神经突起、促进轴突的延伸

(Azmitia et al, 1990)。S100B在哺乳动物的中枢神 经系统内主要由胶质细胞特别是星形胶质细胞分 泌, 其分泌量的增加标志星形胶质细胞被激活, 功 能增强。本实验中用S100蛋白抗体标记S100蛋白的 分布, 主要是S100B蛋白的分布。在大脑运动皮层 的衰老过程中, 神经元、星形胶质细胞和S100蛋白 会出现怎样的形态学变化, 对衰老的敏感是否存在 相互联系? 鉴于此, 本实验以传统的实验动物猫为 研究对象, 采用常规组织学染色和免疫组织化学方 法, 对青年猫和老年猫两年龄组个体的大脑运动皮 层神经元和星形胶质细胞进行形态学观察和定量
比较, 以期探讨神经元、星形胶质细胞在衰老过程 中的相互作用, 为老年个体运动能力下降的神经机 制积累实验资料。

\section{1 材料和方法}

\section{1 实验材料及试剂}

取健康青年猫（2-3 龄, 体重 2-2.5 kg）和 老年猫（10-13 龄, 体重 3.0-3.5 kg）各 4 只, 盐 酸氯胺酮 $(40 \mathrm{mg} / \mathrm{kg}$ 体重) 深度麻醉, 开胸后经左 心室插管升至主动脉, 灌注 $0.9 \%$ 的生理盐水, 至肝 脏发白后灌注含 $4 \%$ 多聚甲醛的 $0.1 \mathrm{~mol} / \mathrm{L}$ 磷酸缓冲 液 $(200 \mathrm{ml} / \mathrm{kg}$ 体重) 进行预固定, 开颖取脑。依猫 脑立体定位图谱（Snider, 1961）定位切取大脑皮层 十字沟后回运动区, 放入 $4 \%$ 多聚甲醛中固定 20-24h, PBS( $0.1 \mathrm{~mol} / \mathrm{L}, \mathrm{pH} 7.4)$ 冲洗, 常规脱水、 透明、石蜡包埋, 作厚 $6 \mu \mathrm{m}$ 的连续冠状切片, 裱片 于 APES 处理的载玻片上置 $37^{\circ} \mathrm{C}$ 温箱内烘干, 每隔 10 张切片取 5 张切片分为五组, 分别用于 Nissl 染 色、S100 免疫组化标记、GFAP 免疫组化标记以及 免疫反应阴性对照实验。Nissl 染色采用 $0.25 \%$ 焦油 紫染液(每 $30 \mathrm{~mL}$ 0.25\%焦油紫水溶液加 10\%醋酸 5 滴)染色。

\section{2 免疫组织化学染色}

石蜡切片脱蜡至水, $3 \% \mathrm{H}_{2} \mathrm{O}_{2}$ 室温孵育 $5 \mathrm{~min}$, 以消除内源性过氧化物酶活性; 蒸馏水冲洗, PBS 浸泡 $5 \mathrm{~min}$; 滴加正常山羊血清, 室温捊育 $10 \mathrm{~min}$, 封闭非特异性反应部位; 倾去血清, 勿洗, 滴加一 抗 (一组为兔抗牛 S100 抗体 $1: 200$; 另一组为兔抗 人 GFAP 抗体 $1: 200), 37^{\circ} \mathrm{C}$ 孵育 $3 \mathrm{~h}$; PBS 冲洗 3 次, 每次 $5 \mathrm{~min}$; 滴加生物素标记二抗 (羊抗兔 IgG 抗血清), $37^{\circ} \mathrm{C}$ 摡育 $10 \mathrm{~min}$; PBS 冲洗 3 次, 每次 $5 \mathrm{~min}$; 滴加辣根酶标记链酶卵白素过氧化物酶工作液— 三抗( $\mathrm{ABC}), 37^{\circ} \mathrm{C}$ 畂育 $10 \mathrm{~min}$; $\mathrm{PBS}$ 冲洗 3 次, 每次 $5 \mathrm{~min} ; \mathrm{DAB}$ 显色, 自来水冲洗, 脱水, 透明, 封片。 阴性对照实验采用正常山羊血清代替一抗,其余步骤 同上。

实验所用 S100 兔抗牛血清及 GFAP兔抗人血清 及配套的免疫组织化学 SP 试剂盒、DAB 购于美国 Labvision 公司。

\section{3 数据采集及统计分析}

分别从 Nissl 染色组、S100 蛋白免疫标记组和 GFAP 免疫标记组中随机选取青年组及老年组染色 切片 (每只猫 20 张), 置于 Olympus BX-51 型显微 
镜下, 用 Image-pro Express 软件观察、计数、测量、 拍照。

Nissl 染色的切片先在低倍镜（x40）下分别圈 出运动皮层的各层次 (I,II,III,V,VI), 然后在高倍镜 ( $\times 400 ）$ 下于所圈出的范围内取 20 个样方（样方 大小为 $50 \mu \mathrm{m} \times 50 \mu \mathrm{m}$ ) 计数神经元数目并换算成细 胞密度值 (cells $\left./ \mathrm{mm}^{2}\right)$ 。免疫组织化学标记切片用上 述类似的方法计数 S100 蛋白免疫阳性细胞和 GFAP 免疫阳性细胞数并换算出阳性细胞密度 (cells/ $\mathrm{mm}^{2}$ ), 测量 GFAP 免疫阳性细胞直径。皮层各层次 的划分以相邻的 Nissl 染色切片为参考标准。

采集的所有数据均以平均值 \pm 标准差 $(\mathrm{X} \pm S D)$ 表 示, 用 One-way Analysis of Variance 或 Two-way Analysis of Variance (ANOVA)比较不同年龄组, 层 次之间的差异显著性。

\section{2 结 果}

\subsection{Nissl 染色结果}

Nissl 染色显示运动皮层为 5 层结构,即分子层 (I )、外颗粒层 ( II )、外雉体层 (III)、内雉体 层 $(V)$ 和多形层 (VI), 内颗粒层 (IV) 在运动 皮层中不存在(图 1-2)。分子层细胞数量最少, 外 雉体层厚度较大, 内锥体层中可见大的雉体细胞和 其顶树突垂直于皮层表面。老年猫和青年猫相比 I 、 II 、 III 层神经元数目无显著变化 $[\mathrm{I}$ : $F_{(1,198)}=0.897, P=0.756$; II $: F_{(1,198)}=2.594, P=0.109$;III: $\left.F_{(1,198)}=1.626, P=0.204\right]$, 但是 $V 、 V I$ 层有显著性差 异 $\left[\mathrm{V}: F_{(1,198)}=5.801, P=0.017\right.$; VI: $F_{(1,198)}=6.492$, $P=0.012$, 神经元密度下降分别为 $6.55 \% 、 5.72 \%$ (表 1)。

\section{表 1 青年猫及老年猫运动皮层中神经元密度比较 $(X \pm S D)$}

Tab. 1 A comparison on density of neurons in the motor cortex of younger and older cats $(X \pm S D)$

\begin{tabular}{ccc}
\hline $\begin{array}{c}\text { 神经元密度 }\left(\text { 细胞数 } / \mathrm{mm}^{2}\right) \\
\text { Density of neurons } \\
\left.\text { (cell number } / \mathrm{mm}^{2}\right)\end{array}$ & $\begin{array}{c}\text { 青年猫 } \\
\text { Young cat }\end{array}$ & $\begin{array}{c}\text { 老年猫 } \\
\text { Old cat }\end{array}$ \\
\hline I 层 Layer I & $130.8 \pm 54.27$ & $127.01 \pm 51.4$ \\
II 层 Layer II & $332.8 \pm 58.2$ & $317.2 \pm 52.3$ \\
III层 Layer III & $268.8 \pm 38.4$ & $258.1 \pm 58.8$ \\
V 层 Layer V & $342.02 \pm 68.5$ & $321.1 \pm 37.6^{*}$ \\
VI层 Layer VI & $332.5 \pm 24.2$ & $314.5 \pm 41.5^{*}$ \\
\hline
\end{tabular}

\section{2 免疫组织化学方法}

青年猫及老年猫运动皮层中各层均见 GFAP 和 S100 阳性反应分布, 其中分子层数量最多, 胞体染 色较深, 多呈棕黄色或黄褐色, 高倍镜下 GFAP 阳 性细胞呈不规则形，并有突起，其胞体及突起内均 可见免疫阳性物质分布（图 3、4）；S100 蛋白分布 于阳性细胞胞浆中, 细胞突起少(图 7、8)。以阳性 细胞平均灰度值为标准, 与青年猫相比, 老年猫免 疫染色着色较深, 免疫反应强度增强 $\left[\mathrm{GFAP}: F_{(1}\right.$, 198) $=463.595 P<0.0001$; S100: $F_{(1,198)}=532.875 P$ $<0.0001]$ （表 2)。统计结果显示老年猫运动皮层中 GFAP-IR 细胞和 S100-IR 细胞密度比青年猫显著上 升[GFAP-IR: $F_{(1,958)}=582.932, P<0.0001$; S100-IR: $F_{(1,958)}=347.529, P<0.0001$ ] (图 3-8，表 3、4）， 这种年龄依赖性影响不因猫的个体而异[GFAP-IR: $F_{(3,958)}=1.75, P=0.155$; S100-IR: $F_{(3,958)}=2.38$, $P=0.095$ ], 但影响大小与层次有关 [层次差异 [GFAP-IR: $F_{(4,958)}=614.271, P<0.0001$; S100-IR: $\left.F_{(4,958)}=227.896, P<0.0001\right]$ 。年龄与层次之间相 互作用 [GFAP-IR: $F_{(1,958)}=23.82, P<0.0001$; S100-IR: $\left.F_{(1,958)}=31.161, P<0.0001\right]$ 。与青年猫 相比, 老年猫运动皮层中 GFAP-IR 密度上升幅度分 别为 I 层 $60.1 \%$ 、II 层 $36.4 \%$ 、III层 $70.9 \%$ 、 V 层 $41.7 \%$ 、VI层 $51.4 \%$; 老年猫运动皮层各层中 S100-IR 细胞密度上升幅度分别为 I 层 $51.1 \%$ 、 II 层 31.2\%、III层 64.9\%、V 层 37.4\%、VI层 31.9\%。 Pearson 分布相关性检验显示, GFAP-IR 细胞和 S100-IR 细胞密度增加存在显著正相关性 $(r=$ 0.546, $P<0.0001$ )。另外，运动皮层中 GFAP-IR 胞 体直径显著增大 $\left[F_{(1,247)}=137.335, P<0.0001\right]$, 平 均增加 23.5\%（表 3); 阴性对照组未见阳性反应。

表 2 青年猫及老年猫运动皮层 GFAP-IR 细胞和 S100-IR 细胞平均灰度值比较 $(\mathrm{X} \pm S D)$

Tab. 2 A comparison on average grey value of GFAP-IR cells and S100-IRcells in the motor cortex of younger and older cats $(\mathrm{X} \pm S D)$

\begin{tabular}{lcc}
\hline $\begin{array}{c}\text { 细胞平均灰度值 } \\
\text { Average grey value of cells }\end{array}$ & $\begin{array}{r}\text { 青年猫 } \\
\text { Young cat }\end{array}$ & $\begin{array}{c}\text { 老年猫 } \\
\text { Old cat }\end{array}$ \\
\hline GFAP-IR & $135.31 \pm 7.71$ & $104.98 \pm 11.79^{*}$ \\
S100-IR & $104.06 \pm 12.43$ & $58.94 \pm 8.81^{*}$ \\
\hline
\end{tabular}




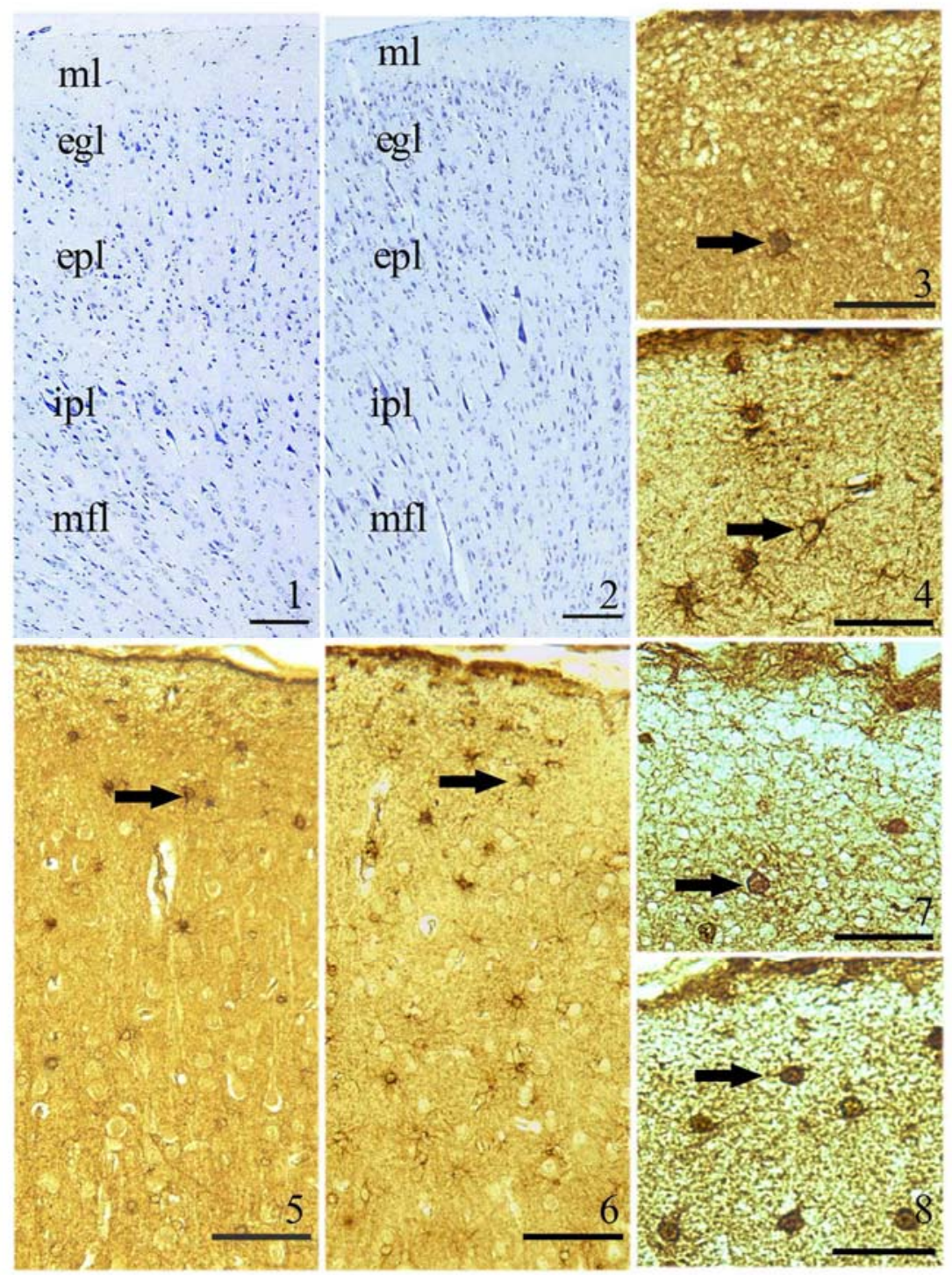

图 1-8 青年猫和老年猫运动皮层分层结构、GFAP-IR细胞和S100-IR细胞

Figs. 1-8 Layers, GFAP-IR cells and S100-IR cells in the motor cortex of young and old cats

$\mathrm{Ml}$ : 分子层; egl: 外颗粒层; epl: 外雉体层; ipl: 内雉体层; mfl: 多形层。 $\mathrm{ml}$ : molecular lamina; egl: external granular lamina; epl: external pyramidal lamina; ipl: internal pyramidal lamina; mfl: multiform layer.

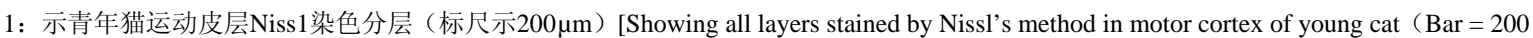
$\mu \mathrm{m})]$;

2: 示老年猫运动皮层Niss1染色分层（标尺示 $200 \mu \mathrm{m}$ ) [Showing all layers stained by Nissl's method in motor cortex of old cat (Bar=200 $\mu \mathrm{m}$ )]; 3: 示青年猫运动皮层分子层 $(\mathrm{ml})$ GFAP-IR细胞(标尺示50 $\mu \mathrm{m}$ )[Showing GFAP-IR cells of molecular lamina (ml) in motor cortex of young cat (Bar=50 $\mathrm{mm})]$;

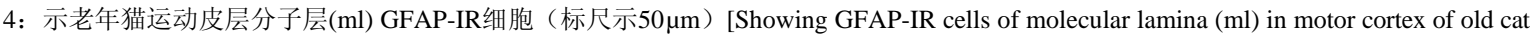
(Bar=50 $\mathrm{mm})]$;

5: 示青年猫运动皮层 I 、II 、III层的GFAP-IR细胞（标尺示 $100 \mu \mathrm{m}$ ） [Showing GFAP-IR cells of I 、II 、III layer in motor cortex of young cat. $(\operatorname{Bar}=100 \mu \mathrm{m})]$;

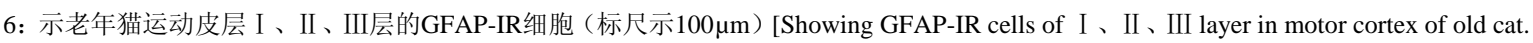

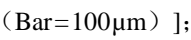

7: 示青年猫运动皮层分子层 $(\mathrm{ml}) \mathrm{S} 100-\mathrm{IR}$ 细胞（标尺示50 $\mu \mathrm{m}$ ） [Showing S100-IR cells of molecular lamina (ml) in motor cortex of young cat

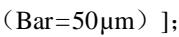

8: 示老年猫运动皮层分子层 $(\mathrm{ml})$ S100-IR细胞 (标尺示50 $\mu \mathrm{m}$ ) [Showing S100-IR cells of molecular lamina (ml) in motor cortex of old cat (Bar $=50 \mu \mathrm{m})$ ]。 
表 3 青年猫及老年猫运动皮层中 GFAP-IR 细胞密度及直径比较

Tab. 3 A comparison on density of GFAP-IR cells in the motor cortex of younger and older cats

\begin{tabular}{lcc}
\hline $\begin{array}{c}\text { GFAP-IR 细胞密度 }\left(\text { 细胞数 } / \mathrm{mm}^{2}\right) \text { 及直径 }(\mu \mathrm{m}) \\
\text { Density }\left(\text { cell number } / \mathrm{mm}^{2}\right) \text { and diameter }(\mu \mathrm{m}) \text { of GFAP-IR cells }\end{array}$ & $\begin{array}{c}\text { 青年猫 Young cat } \\
(\mathrm{X} \pm S D)\end{array}$ & $\begin{array}{c}\text { 老年猫 Old cat } \\
(\mathrm{X} \pm S D)\end{array}$ \\
\hline I 层 Layer I & $220.8 \pm 58.9$ & $355.2 \pm 62.1^{*}$ \\
II 层 Layer II & $211.5 \pm 40.9$ & $288.2 \pm 42.9^{*}$ \\
III层 Layer III & $121.6 \pm 34.4$ & $217.6 \pm 42.4^{*}$ \\
V 层 Layer V & $134.4 \pm 27.8$ & $190.4 \pm 44.6^{*}$ \\
VI层 Layer VI & $112.9 \pm 38.7$ & $169.6 \pm 36.6^{*}$ \\
运动皮层灰质 GFAP-IR 直径 $(\mu \mathrm{m})$ Diameter of GFAP-IR in motor cortex & $6.89 \pm 1.32$ & $8.51 \pm 1.24^{*}$ \\
\hline${ }^{*} P<0.0001$. & &
\end{tabular}

表 4 青年猫及老年猫运动皮层中 S100-IR 细胞密度比较 (细胞数 $/ \mathrm{mm}^{2}$ )

Tab. 4 A comparison on density of S100-IR cells in the motor cortex of younger and older cats (cell number $/ \mathrm{mm}^{2}$ )

\begin{tabular}{lll}
\hline $\begin{array}{l}\text { S100-IR 细胞密度 } \\
\text { Density of S100-IR cells }\end{array}$ & $\begin{array}{l}\text { 青年猫 Young cat } \\
(\mathrm{X} \pm S D)\end{array}$ & $\begin{array}{l}\text { 老年猫 Old cat } \\
(\mathrm{X} \pm S D)\end{array}$ \\
\hline I 层 Layer I & $245.8 \pm 53.1$ & $371.2 \pm 70.4^{*}$ \\
II 层 Layer II & $241.5 \pm 47.4$ & $316.8 \pm 65.5^{*}$ \\
III层 Layer III & $143.6 \pm 34.4$ & $236.8 \pm 45.7^{*}$ \\
V 层 Layer V & $193.6 \pm 30.8$ & $265.3 \pm 36.6^{*}$ \\
VI层 Layer VI & $147.9 \pm 40.9$ & $195.2 \pm 31.8^{*}$ \\
\hline
\end{tabular}

${ }^{*} P<0.0001$

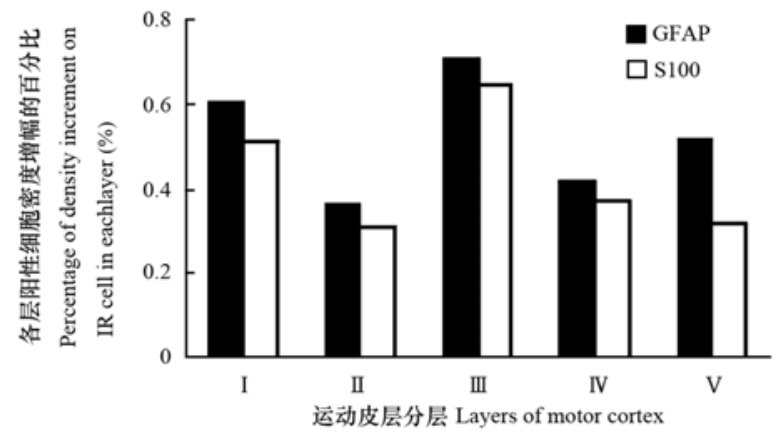

图 9 老年猫运动皮层各层与青年猫相比 GFAP-IR 细胞和 S100-IR 细胞密度增加百分比

Fig. 9 A comparison on percentage of density increment on GFAP-IR cells and S100-IR cells in the motor cortex of younger cats and older cats

S100-IR 细胞密度增加幅度与 GFAP-IR 细胞增加幅度具有显著的相关性 (Pearson 分布相关性检验 $r=0.929, P=0.023$ )。

The density increment of GFAP-IR cells and S100-IR cells was correlated (Correlation test of Pearson distribution $r=0.929, P<0.023$ ).

\section{3 讨 论}

近些年对衰老机制的研究普遍认为衰老导致了 脑形态和功能的变化 (Erraji-Benchekroun et al, 2005), 如人小脑蚓体(Sjöbeck et al,1999)、猴大脑皮 层(Smith et al,2004)神经元数量的减少, 神经元结构 的改变等。有些形态学研究显示, 衰老过程中神经元
丢失并非是普遍发生的现象，而是与区域、层次及细 胞类型有关(Morrison \& Hof, 1997; Markham et al, 2007)。也有学者(Peters, 2002)认为人类和灵长类动物 的大脑皮层衰老过程中, 神经元没有明显变化。通过 对猫运动皮层常规染色观察统计, 结果显示: 与青年 猫相比, 老年猫运动皮层神经元密度在 I 、 II 、III 层中无显著性差异，而 V、VI层神经元数目较青年 猫显著性下降（表 1)。运动皮层的第 V、VI 层神经 元是主要的运动传出神经元, 第 $\mathrm{V}$ 层内分布有巨大 的 Betz 细胞, 其发出的轴突为锥体系的重要组成部 分, 与随意运动和精细运动有密切关系。因此, 衰 老过程中运动皮层第 V、VI层神经元数目的显著减 少很可能是导致老年个体随意运动和精细运动调控 能力降低的重要原因。

免疫组织化学方法研究发现, 在哺乳动物衰老 或病理状态下人大脑皮质（Korolainen et al, 2005）、 大鼠小脑皮质 (Sabbatini et al,1999) 大鼠视神经 (Cavallotti et al, 2003) 及人的视神经 (Cavallotti et al, 2002）中星形胶质细胞数目增多, 阳性反应增强, 胞体直径增大, 突起增多。本实验以 GFAP 标记星 形胶质细胞, 结果显示老年猫运动皮层中星形胶质 细胞也出现显著增生, 突起增多延长, 免疫反应性 增强, 表明老年猫运动皮层星形胶质细胞的功能增 强, 与前人在衰老大鼠大脑皮质、小脑皮质以及人 视网膜的研究结果一致。

S100B作为神经元和星形胶质细胞相互联系的 中介之一, 具有广泛的生物学活性, 作用于神经元 和周围环境, 涉及促进轴索生长、神经胶质细胞增 殖、神经元的分化以及钻自动调节动态平衡

(Zimmer et al,1995)。有学者提出了脑老化的细胞 内 $\left[\mathrm{Ca}^{2+}\right] i$ 平衡自体失衡假说, 认为细胞内正常的生 理量的 $\left[\mathrm{Ca}^{2+}\right] i$ 是维持细胞正常功能必需的, 但是 $\left[\mathrm{Ca}^{2+}\right] i$ 会随着年龄的增加而增加, 持续升高会对神 经元产生毒害，导致其死亡，从而导致帕金森、阿 
尔采默氏病的发生。老年个体的S100表达增强可调 节神经细胞外 $\mathrm{Ca}^{2+}$ 内流, 降低Ca${ }^{2+}$ 超载对神经细胞 的毒害 (Khachaturian, 1989; Raza et al, 2007)。有 研究发现, 在衰老过程中, 大鼠的大脑皮层的S100 的mRNA及其表达产物增加 (Linnemann \& Skarsfelt, 1994)。本实验亦观察到类似的现象, 即猫大脑运 动皮层中S100-IR数量在衰老过程中明显增加。由此 我们推测S100蛋白表达量的增加可以提高神经元 存活率, 维持突触形态, 缓解老年猫神经元的衰老。

大脑皮层的不同层次具有不同的功能，一般认 为第 II 、III、IV层主要接受外界传入皮层的投射纤 维, 负责传入信息的加工和整理, 但在运动皮层中 没有第IV层 (Jiang, 2002); V、VI层主要是运动信 息的投射传出部位。在实验中我们观察到负责皮层 信息传出的 V、VI两层的GFAP - IR 增加幅度为 $51.4 \%$ 和 $41.7 \%$, 而在这两层中的神经元密度出现 显著下降。Araque 等发现, 由突触相关的胶质细 胞和突触前及突触后神经元构成了一种三向突触

(Araque et al,1999)。体外培养研究发现, 星形胶 质细胞可以使突触活动大幅提高, 使成熟的、功能 完善的突触数量大幅增加 (Pfrieger \& Barres, 1997)。由此我们推测星形胶质细胞数量增多、功能 增强以维持衰老神经元的活性以及神经元之间的 通讯联系, 增强突触活动, 弥补了运动皮层因衰老 导致的对运动调控能力的下降。同时我们也观察到 负责传入信息加工和整理的传入皮层GFAP-IR增加 幅度较传出皮层大, 其中III层的星形胶质细胞数量 增加幅度最大, GFAP-IR增加幅度高达 $78.1 \%$ 。 Laskawi et al (1997) 认为, 通过对面神经和三叉神 经的外科损伤能够引发大脑运动皮层的传入层

\section{参考文献:}

Araque A, Parpura V, Sanzqiri RP, Haydon PG. 1999. Tripartite synapses: Glia, the unacknowledge partner[J]. Trends Neurosci, 22(5): 208-215.

Azmitia EC, Dolan A, Whitaket-Azmitia PM. 1990. S100B but not NGF, EGF, insaline or calmodulin is a CNS serotonergic growth factor[J]. Btain Res, 516: 354-356.

Cavallotti C, Artico M, Pescosolido N, Leali FM, Feher J. 2003. Age-related changes in rat optic nerve: Morphological studies[J]. Can $J$ Ophthalmol, 32(1): 12-16.

Cavallotti C, Pacella E, Pescosolido N, Tranquilli-Leali FM, Feher J. 2002. Age-related changes in the human optic nerve[J]. Can J Ophthalmol, 37(7): 389-394.

Dickstein DL, Kabaso D, Rocher AB, Luebke JI, Wearne SL, Hof PR. 2007. Changes in the structural complexity of the aged brain[J]. Aging Cell, 6(3): 275-284.

Erraji-Benchekroun L, Underwood MD, Arango V, Galfalvy H, Pavlidis P,
（ I 、II 、 III）星形胶质细胞标志物GFAP和S100B 的免疫反应增强。据此我们认为猫运动系统中其他 部分, 如小脑、脑干、脊髓、肌肉功能衰退的皮层 外因素可能触发大脑运动皮层传入层出现年龄相 关的形态学变化。这与Laskawi et al (1997) 的研究 结果相一致。这种具体的触发机制也值得进一步探 讨。

中枢神经系统中的S100B主要由星形胶质细胞 分泌, S100B本身对星形胶质细胞的增殖也有促进 作用，两者之间形成了正反馈作用(Selinfreund et al, 1991)。本实验观察到GFAP-IR和S100-IR细胞在运 动皮层各层中均有分布。数据用SPSS软件分析两种 阳性细胞密度分布具有线性相关 $(r=0.546 P<$ $0.0001)$, GFAP-IR 和S100-IR细胞在猫运动皮层各 层中增幅也具有线性相关性 $(r=0.929 P<0.023)$ 。 这种相关性可能正是 $\mathrm{S} 100 \mathrm{~B}$ 和星形胶质细胞之间正 反馈作用的体现。

有研究认为超过正常生理量的神经胶质细胞和 S100 蛋白对神经系统也有不利影响 (Sheng et al, 1996)。神经胶质细胞表达过量时会导致神经系统的 功能紊乱 (Van Eldik \& Wainwright, 2003)。过量增生 的胶质细胞能够阻碍轴突再生, 排挤周围神经元, 影响突触传递的效率, 干扰神经元环路功能 (Ridet et $\mathrm{al}, 1997$ )。随着衰老过程的推进, 激活的星形胶质细 胞密度和 S100B 浓度会不会持续增长, 是否会对运 动皮层的调控能力产生负面影响, 这值得进一步探 讨。

致谢: 感谢中国科学技术大学视觉研究实验室 为我们提供珍贵的老年猫材料!

Smyrniotopoulos P, Mann JJ, Sibille E. 2005. Molecular aging in human prefrontal cortex is selective and continuous throughout adult life[J]. Biol Psychiatry, 57(5): 549-558.

Jessen KR. 2004. Glial cells[J].The International Journal of Biochemistry \& Cell Biology, 36: 1861-1867.

Jiang WH. 2002. Neuroanatomy [M]. In: Shanghai: Fudan University Press, 320-321. [蒋文华. 2002. 神经解剖学. 上海: 复旦大学出版社, 320-321.]

Khachaturian ZS. 1989. The role of calcium regulation in brain aging: reexamination of a hypothesis[J]. Aging (Milano), 1(1): 17-34.

Kirchhoff F, Dringen R, Giaume C. 2001. Pathways of neuron: Astrocyte interactions and their possible role in neuroprotection[J]. Eur Arch Psychiatry Clin Neurosci, 251 (4): 159-169.

Komatsu M, Waguri S, Chiba T, Murata S, Iwata J, Tanida I, Ueno T, Koike M, Uchiyama Y, Kominami E, Tanaka K. 2006. Loss of autophagy in 
the central nervous system causes neurodegeneration in mice[J]. Nature, 441(7095): 819-820.

Korolainen MA, Auriola S, Nyman TA, Alafuzoff I, Pirttilä T. 2005. Proteomic analysis of glial fibrillary acidic protein in Alzheimer's disease and aging brain[J]. Neurobiol Dis, 20(3): 858-870.

Laskawi R, Rohlmann A, Landgrebe M, Wolff JR. 1997. Rapid astroglial reactions in the motor cortex of adult rats following peripheral facial nerve lesions[J]. Eur Arch Otorhinolaryngol, 254(2): 81-85.

Linnemann D, Skarsfelt T. 1994. Regional changes in expression of NCAM, GFAP, and S100 in aging rat brain[J]. Neurobiol Aging, 15: 651-665.

Morrison JH, Hof PR. 1997. Life and death of neurons in the aging brain[J]. Science, 278: 412-419.

Markham JA, Morris JR, Juraska JM. 2007. Neuron number decreases in the rat ventral, but not dorsal, medial prefrontal cortex between adolescence and adulthood[J]. Neuroscience, 144(3): 961-968.

Peters A. 2002. Structural changes in the normally aging cerebral cortex of primates [J]. Prog Brain Res, 136: 455-465.

Pfrieger FW, Barres BA. 1997. Synaptic efficacy enhanced by glial cells in vitro[J]. Science, 277(5332): 1684-1687.

Raza M, Deshpande LS, Blair RE, Carter DS, Sombati S, DeLorenzo RJ. 2007. Aging is associated with elevated intracellular calcium levels and altered calcium homeostatic mechanisms in hippocampal neurons[J]. Neurosci Lett, 418(1): 77-81.

Ridet JL, Malhotra SK, Privat A, Gaqe FH. 1997. Reactive astrocytes: cellular and molecular cues to biological function[J] . Trends Neurosci, 20(12): 5770-5777.

Sabbatini M, Barili P, Bronzetti E, Zaccheo D, Amenta F. 1999. Age-related changes of glial fibrillary acidic protein immunoreactive astrocytes in the rat cerebellar cortex[J]. Mechanisms of Ageing and Development,
108: $165-172$.

Sailer A, Dichgans J, Gerloff C. 2000. The influence of normal aging on the cortical processing of a simple motor task[J]. Neurology, 55(7): 979-985.

Selinfreund RH, Barger SW, Pledger WJ, Van Eldik LJ. 1991. Neurotrophic protein S100 beta stimulates glial cell proliferation[J]. Proc Natl Acad Sci U S A, 88(9): 3554-3558.

Sheng JG, Ito K, Skinner RD, Mrak RE, Rovnaqhi CR, Van Eldik LJ, Griffin WS. 1996. In vivo and in vitro evidence supporting a role for the inflammatory eytokine interleukin-1 as a driving force in Alzheimer pathogenesis[J]. Neurobiol Aging, 17: 761-766.

Sjöbeck M, Dahlén S, Englund E. 1999. Neuronal loss in the brainstem and cerebellum: Part of the normal aging process? A morphometric study of the vermis cerebelli and inferior olivary nucleus[J]. $J$ Gerontol A Biol Sci Med Sci, 54(9): B363-368.

Smith DE, Rapp PR, McKay HM, Roberts JA, Tuszynski MH. 2004. Memory impairment in aged primates is associated with focal death of cortical neurons and atrophy of subcortical neurons[J]. J Neurosci, 24(18): 4373-4381.

Snider RS. 1961. A stereotaxic Atlas of the Cat Brain [M]. Chicago: The University of Chicago Press.

Van Eldik LJ, Wainwright MS. 2003. The Janus face of glial-derived S100B: Beneficial and detrimental functions in the brain[J]. Restor Neurol Neurosci, 21(3-4): 97-108.

Ward NS. 2006. Compensatory mechanisms in the aging motor system [J]. Ageing Res Rev, 5(3): 239-254.

Zimmer DB, Cornwall EH, Landar A, Song W. 1995. The S100 protein family: History function, and expression[J]. Brain Res Bull, 37(4): 417-429.

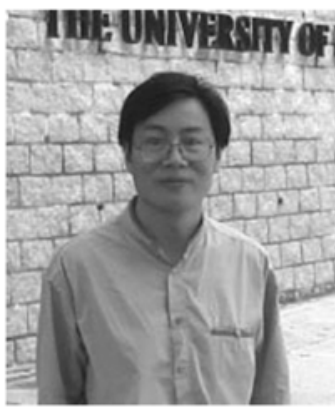

周荣家教授

\section{本刊编委周荣家教授简介}

周荣家, 男, 1961 年生, 武汉大学生命科学学院教授, 博士生导师。主 要从事发育遗传学研究。1978-1982、1983-1986 年分别在四川农业大学取 得学士和硕士学位, 1989-1992 在华西医科大学取得博士学位, 1992-1994 在武汉大学生物 (遗传) 学博士后流动站从事博士后研究。曾对美国加州大学 旧金山分校 (UCSF)、Louisiana 州立大学、澳大利亚 Latrobe 大学、国立新 加坡大学、法国人类遗传学研究所 (CNRS)、巴斯德研究所 (Pasteur)、香港 大学等分别进行过访问和合作研究。1996 年起任武汉大学教授, 1998 年起被 评为博士生导师。任国际刊物 Zebrafish 编委、核心刊物《遗传学报》、《遗传》 和《动物学研究》编委、中国遗传学会动物遗传学专业委员会委员、中国空间 生命专业委员会委员、国家科技奖励评审专家等职。2006 年入选教育部 “新 世纪优秀人才计划”。2006 年享受政府特殊津贴。

研究兴趣主要集中在早期胚胎发育、分化和器官形成过程中所涉及的众多基因的时空表达与调控。通 过进化时空和多个模式物种的比较研究, 以及功能基因组, 转录组和蛋白质组等多种现代生命科学手段, 研究基因-发育-进化等生命本质, 尤其关注胚胎发生, 性别决定, 细胞凋亡等生命活动。曾获湖北省自然科 学二等奖、湖北省青年科技奖、中国遗传学会李汝祺优秀动物遗传学论文奖等多项奖励。主持过 973、863、 国家自然科学基金、教育部重点项目、霍英东青年基金等多项国家、省市科研课题以及国际人类前沿科学 组织 (HFSPO) 研究和中法先进研究计划 (PRA) 项目。发表论文 100 余篇, 其中发表在 Nucleic Acids Res、 BBA Mol Cell Res、Carcinogenesis、Biol Reprod、Reprod BioMed Online、J Cell Biochem、J Exp Zool 等 SCI 刊物论文 37 篇。主译著作《发育原理》和编著《转基因动物技术与应用》等。 